\title{
A practical guide to behavioral theory-driven statistical development of quality and safety improvement program in health care
}

\begin{abstract}
The health care field has adopted many process improvement methodologies from other disciplines and industries to ensure quality and patient safety. However, there is still a great need for a well-structured and easy-to-follow methodology that focuses on changing health care professionals' (HCPs) behaviors. Using hand hygiene among intensive care unit HCPs as an illustrative example, the authors provide a practical guideline for such a methodology. Based on Fishbein \& Ajzen ${ }^{1}$ theory of planned behavior and Hornik \& Woolf ${ }^{2}$ message design strategy, this methodology identifies key factors, determines their intention for the behavior at hand, runs a cross-sectional survey on the beliefs and behavioral intention, and uses statistical analysis to identify a manageable number of target beliefs that will be the basis for program development. In addition, we introduce ways to develop a successful program that is based on this information.
\end{abstract}

Keywords: healthcare provider behavior, theory of planned behavior, statistical approach to program development, quality of care, patient safety, hand hygiene
Volume I Issue I - 2014

\author{
Heon Jae Jeong,' Minji Kim² \\ 'Department of Health Policy and Management, Bloomberg \\ School of Public Health, Johns Hopkins University, USA \\ ${ }^{2}$ Annenberg School for Communication, University of \\ Pennsylvania, USA
}

Correspondence: Heon-Jae Jeong, Department of Health Policy and Management, Johns Hopkins University, Bloomberg School of Public Health, 624 North Broadway, Rm. 455, Baltimore, MD, 21 205, USA, Tel 410-955-5315, Email hj957।@gmail.com

Received:September 10, 2014 | Published: September 18, 2014

\section{Introduction}

In order to improve quality and patient safety, health care organizations around the world have launched an immeasurable number of projects. To expedite this improvement, many of them have borrowed well-recognized techniques from other industries or fields, so-called "translocational research." For instance, the policy of limiting the number of consecutive work-hours was adopted from the airlines and trucking industries. ${ }^{3}$ Moreover, for more complicated and large-scale projects like clinical process redesign, more comprehensive methodologies such as Lean Six Sigma or Total Quality Management have been borrowed from business, management and even manufacturing, and proven effective in health care., ${ }^{4,5}$

However, such translocational application of methodologies customarily targets system or process improvement; few are intended to change the behavior of individual health care professionals (HCPs). This is regrettable because several simple but critical behaviors can enhance quality and safety, such as verbal order read back, doublechecking patient ID when administering medication, and having a timeout before beginning a surgery. Even a large systems improvement contains several parts that require HCPs' behavioral changes. To illustrate, a hospital-wide implementation of Computerized Prescriber Order Entry system requires physicians to change their behavior, something that is expected to take quite a long time. Indeed, there is a huge unmet need for a proven methodology to tap into HCPs' minds and expedite their behavioral modification.

In search of such a methodology, we must keep in mind that each organization or work unit possesses its own culture and behavioral pattern, which must be factored into any behavioral change program; simply trying to replicate the 'best practice' or 'success story' of other organizations is likely to be ineffective at best and a failure at worst. ${ }^{6}$

This article, introduces a methodology, drawn from the fields of social psychology and communication that is designed to effect HCPs' behavioral change. It also offers how to identify the underlying beliefs on the behavior held by each HCP and even social pressure from the culture where they work.

In order to keep this guide easy to follow, we have refrained from describing the theoretical background and the statistical analysis techniques in detail. Instead we suggest step-by-step activities intended to solve a fictional but highly plausible problem-low compliance in hand hygiene behavior of resident physicians working in the Intensive Care Units (ICUs) of a hospital (Box 1).

\section{Case (Problem) Description}

In ICUs of hospital A, the most important rule to protect patients from infection is for HCPs to wash their hands with either an alcohol-based rub or soap and water before and after seeing a patient. Using the 'secret shopper' method, hospital A found that resident physicians' hand hygiene compliance was below $30 \%$, significantly lower than that of nurses $(90 \%)$, and staff physicians (85\%).

The chief physician of ICUs and even the top executives of the hospital are paying much attention to bringing residents' compliance with hand hygiene behavior at least to the level of nurses and staff physicians. You, as hospital A's quality and safety leader, have been asked to design a program to solve this problem. However, your experience in improving hand hygiene is limited to educating HCPs that infections can be fatal to patients. Despite the regular provision of such education, it has not been be very effective among resident physicians, so you are at a loss as to what else you can do.

Box 1 Scenario.

\section{Background theory and behavioral change strategy}

To provide you with a step-by-step guide, we begin with the wellknown Theory of Planned Behavior, or TPB,${ }^{1,7}$ which will serve as the framework of this guide. The model in Figure 1 is simplified from the original TPB and adapted to solve the problem of residents' poor compliance with hand hygiene. Some of you might think that 
the model resembles one of the most frequently utilized root cause analysis tools, the fishbone diagram, but by looking deep into the components, it is easy to see that this model is solely to change or modify behavior.

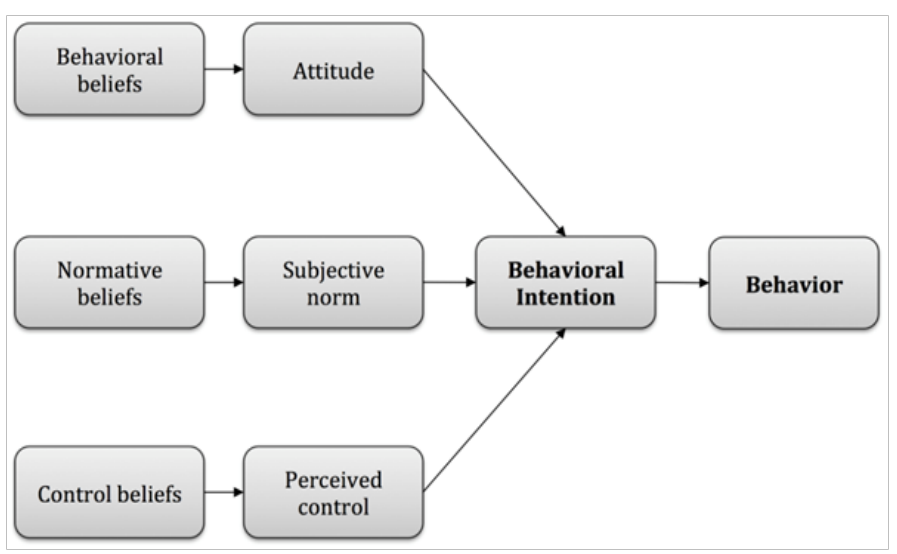

Figure I Theory of planned behavior.'

The purpose of this approach is to understand why some people perform (or fail to perform) the target behavior (the box farthest to the right), in our case, residents' hand hygiene behavior, and change that behavior. TPB assumes that the most important predictor of the behavior is a person's intention to perform that behavior. Without intention, a person is less likely to perform the desired behavior.

The model also assumes that the intention is the combined result of one's attitude toward the behavior, the ways in which others encourage or discourage that behavior (subjective norm) and the ways in which situations around the person facilitate or hamper the person from performing that behavior (perceived control). The relative importance of these three determinants of behavior (attitude, subjective norm, perceived control) will depend on the behavior and population, and therefore it is important to understand how each determinant predicts behavioral intention for a given situation. However, to make changes among 'non-intenders,' determining which of the three is the most important is only part of the work; ultimately, one should understand all of the underlying drivers - behavioral, normative and control beliefs of the given population (Figure 1). ${ }^{8}$

Behavioral beliefs, also known as outcome beliefs, are what one expects from performing the behavior. To illustrate, one might think that "washing hands frequently can save patients from hospitalacquired infection"; in the meantime, one can think that "hand hygiene makes the skin of one's hands too dry and sore." As you might guess the more positive outcomes that are associated with the desired behavior, the more likely one is to perform it. In contrast, normative beliefs are the way in which one perceives the influence on others of performing the behavior. For example, residents might be influenced by the perception that their fellow residents, nurses and supervising physicians encourage or discourage their performance of the behavior (e.g., "my colleague residents want me to conduct hand hygiene"). Lastly, control beliefs include situations that facilitate or hamper performing the desired behavior. One of the easy examples would be "the alcohol-based rub dispenser in the patient's room is frequently empty."

There are a large number of behavioral, normative and control beliefs behind performing a specific behavior in a specific place where the behavior is influenced by the organizational or unit-based culture.
Therefore, ways to change HCPs behavior are

i) To reveal deeply held beliefs that seldom surface

ii) To identify key beliefs that are most significantly related to performing the behavior

iii) To develop programs that can change the selected key beliefs and thus bring the most impact to one's performing the behavior. This guide, therefore, presents a practical approach to those three steps using the hand hygiene case shown in box 1 as an example. The authors have created a fictitious dataset as an illustrative example to walk through the belief selection processes. The items are based on a previous qualitative study. ${ }^{9}$ The associated statistics in the following tables are fictitious, but does well reflect the findings from previous study as well as the authors' years-long experience working with healthcare professionals in ICU environments.

\section{A Step-by-step guide}

\section{Reveal the salient beliefs}

The first step is defining the target behavior. The more specific it is, the more accurately this step will identify beliefs that will change the behavior. The definition should incorporate action, target, context and time. ${ }^{10}$ Based on the case in box 1 , we define the target behavior as "Residents washing hands using alcohol-based rub or soap and water before and after seeing a patient in ICUs in the next week." A precise description of behavior is crucial since we will collect beliefs related to that behavior.

With the defined behavior, we run a series of elicitation interviews to learn what relevant beliefs the target population possesses. An elicitation interview is semi-structured (Table 1) and open-ended, usually conducted on at least 15 to 20 respondents. However, the absolute number of interviews is of not too much importance. The purpose of the interviews is to reveal all the salient beliefs until more new beliefs emerge. From the authors' experience, beliefs among a limited population like HCPs working in an ICU require only 1012 interviews until reaching belief saturation. It is desirable to have about half of the interviewees having already performed or intending to perform the behavior, and the other half having not performed well. ${ }^{11}$ The decision of who should be interviewed should be left to the discretion of the project manager, with input from people in the best position to have observed behavior or the target population; in this case, nurses and senior physicians. The rationale for the intender vs. non-intender mix will be described in the next step.

Though the focus group interview is known to be efficient and time-saving, the one-on-one setting is suggested to prevent any group dynamics effect and to facilitate the interviewees to express underlying beliefs. Table 1 shows some sample questions. Asking probing questions is a good way to elicit beliefs that are not readily accessible but may still be influential.

Once we have reached saturation, it is time to analyze the interview results to identify the most common beliefs held by the interviewees. The interviews are usually audio-recorded with participants' consent, so typing them into computer is preferred. Either content analysis software or even a simple spreadsheet with manual analysis suffices to organize the interviewees' beliefs. A researcher can group different terms and expressions that refer to the same outcome (e.g., "makes skin sore" and "skin irritation"). Then, beliefs are ranked by their frequency of mention, until the chosen beliefs account for at least $75 \%$ 
of responses. ${ }^{1}$ Through interview and content analysis, we generated a list of the 12 beliefs about hand washing that were held by residents working in ICUs (Table 2). ${ }^{9}$

The beliefs elicited through the interviews and following content analysis will be fed into formative survey in the next step, so that quantitative information on the associations among behavior, behavioral intention, and the underlying beliefs will be analyzed.

\section{Identify key beliefs by formative survey}

While we revealed the 12 most frequently mentioned beliefs, designing a program that covers all the beliefs will be likely to fail because of a lack of focus. Furthermore, if elicitation interviews revealed tens of salient beliefs, it would be impossible to develop a program addressing all of them. This step therefore describes how we can identify key beliefs that must be addressed in program development.

Table I Examples of elicitation interview questions

\begin{tabular}{ll}
\hline Attitude (behavioral beliefs) & $\begin{array}{l}\text { What are the advantages/disadvantages of washing hands before and } \\
\text { after seeing a patient, using alcohol-based rub or soap and water in } \\
\text { ICUs in the next week? } \\
\text { What would you expect from your washing your hands...? }\end{array}$ \\
$\begin{array}{ll}\text { Social Norm (normative beliefs) } & \text { Who is influential on your washing your hands...? } \\
\text { Perceived Behavioral Control } & \text { What would you expect from your washing your hands...? } \\
\text { (control beliefs) } & \text { What makes it easy or difficult for you to wash your hands...? }\end{array}$ \\
\hline
\end{tabular}

Table 2 Elicited relevant beliefs

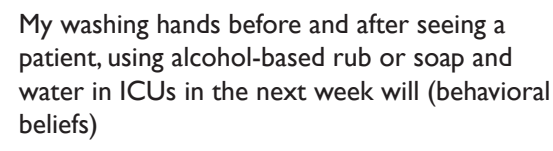
patient, using alcohol-based rub or soap and water in ICUs in the next week will (behavioral beliefs)

Those who think I should wash my hands... (normative beliefs)

Perceived Behavioral Control (control beliefs)

\author{
Protect patients from infection \\ Protect resident (self) from infection \\ Makes skin too dry and sore on resident's hands \\ Consumes too much time (when using soap and water) \\ Other residents \\ Staff physicians (seniors) \\ Nurses \\ Mentally alert patients
}

When seniors do not wash their hands (residents were afraid of challenging their seniors by washing their own hands while the seniors do not)

Emergency Situation (e.g., patient needs cardiopulmonary resuscitation)

Alcohol-based rubs are not around

Forgetting to wash one's hands
First, we develop a survey whose main items to be measured are intention to perform the behavior, and the beliefs we found in step 1 . Intention is measured on a four-point Likert-type scale from "definitely will not" (1) to "definitely will" (4), because we will dichotomize the respondents into intenders and non-intenders. Beliefs are commonly measured on a five-point Likert-type scale from "strongly disagree" (-2) to "strongly agree" (2). ${ }^{12}$ For example, "Washing my hands before and after seeing a patient, using alcohol-based rub or soap and water in ICUs in the next week will protect patients from infection" (behavioral belief); "Other residents think that I should wash my hands before and after seeing a patient, using alcohol-based rub or soap and water in ICUs in the next week" (normative belief); "I will experience an emergency situation" (control belief). We must reverse the scoring scheme for beliefs that are expected to have deterred the target behavior. To illustrate, for an item like "Washing my hands before and after seeing a patient, using alcohol-based rub or soap and water in ICUs in the next week makes my skin dry and sore" the answer "strongly disagree" should be considered as if "strongly agree," and scored -2 rather than +2 in analysis, so that high score consistently indicates beliefs favorable toward the target behavior.

In a small-scale project targeting a certain population (ICU working residents), a survey could ideally be administered to the entire target population. However, even at a single ICU, administering survey to all HCPs is practically impossible, considering that their work hour is already overloaded. Therefore, in reality we would naturally gather survey data from a sample of the whole target population.

After all surveys have been returned, we can calculate the sample correlation coefficients between each belief strength (measured on a scale of -2 to +2 ) and behavioral intention (measured on a scale of 1 to 4$)$. We have 12 beliefs, so that 12 correlation coefficients in total will be calculated (so called zero-order correlation coefficients). We 
would also focus on the percentage of people who strongly agreed (belief score +2 ) with each belief between intenders (intention score 3 or 4 ) and non-intenders (intention score 1 or 2). Table 3 displays these information; as mentioned earlier, the dataset shown in the table is an illustrative example. Although the authors have used both STATA and Microsoft Excel in our analyses and they produced the same zeroorder correlation coefficients, any other appropriate software can be used to obtain these statistics.

a. Using Table 3, we select a few target beliefs to be used in program development by utilizing the criteria suggested by Hornik \& Woolf $^{2}$ The belief should be significantly related to the behavioral intention (significant correlation)

b. There should be enough "room for change" for beliefs. In Table 3, the larger the difference in percentage of whom answered "strongly agree" with a belief between intenders and non-intenders, the more room for change is expected

c. Whether a belief can be modified through the program we will develop; if not, the beliefs should not be chosen.

We will apply Hornik \& Woolf ${ }^{2}$ first two criteria in this section, and demonstrate the selection of target beliefs. Among the four behavioral beliefs, all of them show a significant correlation with behavioral intention, so they all pass the first criterion. However, regarding room for change, there are noticeable differences across beliefs; while "Protect resident oneself from infection," "Makes skin too dry and sore on resident's hands (reversed)," and "Consumes too much time when using soap and water (reversed)" are held relatively much smaller portion of non-intenders when compared to intenders, "Protects patients from infection" is widely held among even the nonintenders. Therefore, this belief, even with its significant correlation, should not be selected. Among the normative beliefs, "staff physicians" and "mentally alert patients" show significant correlation. Both beliefs are held by less than half of the non-intenders, while $75 \%$ and $81 \%$ of intenders strongly hold these two beliefs. Therefore, these two beliefs meet the first two criteria.

Table 3 Statistical analysis on beliefs and behavioral intention

\begin{tabular}{|c|c|c|c|c|c|}
\hline \multirow[b]{2}{*}{ Beliefs } & \multirow[b]{2}{*}{ Correlation $\mathbf{r}$} & \multicolumn{2}{|c|}{ Mean belief } & \multicolumn{2}{|c|}{ \% Strongly agree } \\
\hline & & $\begin{array}{l}\text { Non- } \\
\text { intender }\end{array}$ & Intender & $\begin{array}{l}\text { Non- } \\
\text { intender }\end{array}$ & Intender \\
\hline Protects patients from infection & $0.16 * *$ & 1.51 & 1.63 & 67 & 73 \\
\hline Protects resident (self) from infection & $0.26 * *$ & 0.38 & 0.97 & 31 & 60 \\
\hline Makes skin too dry and sore on residents' hand ( $r$ ) & $0.34 * *$ & 0.28 & 1.07 & 22 & 59 \\
\hline $\begin{array}{l}\text { Consumes too much time (when using soap and } \\
\text { water) ( } r \text { ) }\end{array}$ & $0.25 * *$ & 0.81 & 1.30 & 40 & 73 \\
\hline \multicolumn{6}{|l|}{ Normative beliefs } \\
\hline $\begin{array}{l}\text { Other residents think I should follow hand hygiene } \\
\text { guideline }\end{array}$ & 0.07 & 0.39 & 0.70 & 38 & 60 \\
\hline $\begin{array}{l}\text { Staff physicians } \\
\text { (seniors) think I should follow hygiene guideline }\end{array}$ & $0.37^{* * *}$ & -0.20 & 1.10 & 38 & 75 \\
\hline Nurses think I should follow hand hygiene guideline & 0.06 & 0.55 & 0.74 & 56 & 67 \\
\hline $\begin{array}{l}\text { Mentally alert [patients think I should follow hand } \\
\text { hygiene guideline }\end{array}$ & $0.18 * *$ & 0.92 & 1.32 & 44 & 81 \\
\hline \multicolumn{6}{|l|}{ Control beliefs } \\
\hline Senior do not wash their hands $(r)$ & $0.37 * *$ & -0.30 & 1.10 & 80 & 92 \\
\hline Emergency situations (e.g., patient needs CPR) (r) & $0.26 * * *$ & 0.33 & 1.00 & 30 & 60 \\
\hline Alcohol-based rubs are not around ( $r$ ) & $0.24 * *$ & -0.70 & 0.40 & 48 & 76 \\
\hline I forget to wash my hands ( $r$ ) & $0.20 * *$ & -0.35 & 0.29 & 16 & 36 \\
\hline
\end{tabular}

$(r)$ : reversely coded items, correlation means how each belief and intention is correlated, ${ }^{* *}: \mathrm{p}<.0 \mathrm{I},{ }^{* * *}: \mathrm{p}<.00 \mathrm{I}$

Lastly, all the control beliefs are significantly correlated with intention, and held by significantly fewer non-intenders than intenders, which makes all those beliefs meet the first two criteria. The third criterion is whether a selected belief can be used in developing a program. We will use the control belief about "emergency situations" as an example. It is understandable that it may be almost impossible to adhere to hand hygiene guidelines in an emergency. Moreover, ICU is one of the units where emergency situations occur the most frequently in a hospital. It would be impossible to design a program that would reduce the number of emergency situations in ICU, and it would be unrealistic to expect residents to make hand hygiene a higher priority than saving a patient's life. Therefore, while this belief has met the first two criteria, it will not be a good basis for a program.

The normative belief that "mentally alerts patients think I should follow hand hygiene guideline" is in similar position. Residents would naturally feel more pressured to wash their hands when patients will know whether or not they do it. However in ICUs, most patients are not expected to be conscious and paying attention. Moreover, it is unacceptable for residents to wash their hands only when the patient is watching. Therefore, this belief is not a sound basis for a program. By 
testing each belief through these criteria, we arrive at seven key items to focus on in developing a program to improve residents' compliance with hand hygiene behavior. The gray items in Table 3 are the items which will be used in the next step.

\section{Develop an effective program using selected beliefs}

Now, we develop a program, "Improving residents' hand hygiene compliance in ICUs" focusing on the seven key beliefs found above. It can't be stressed enough that while the way to find key beliefs are well established as shown above, developing a program is a highly creative task requiring program developer's in-depth understanding on target place's resource availability, general compliance to a quality and safety improvement program of the target population, even the overall culture of the place; the expected support level from senior physicians and executives should not be ignored. ${ }^{6}$

Here, we suggest a fictional example program by assuming that hospital and senior executives are willing to provide financial support to post reminders and to purchase more alcohol-based hand rubs and food for occasional lunch meetings. The following program example is derived from the above analysis; since one program components can cover several beliefs (and vice versa) the (Table 4) below is arranged to reflect that flexibility.

It is important to increase both self-efficacy (perceived ease of performing the target behavior) and response efficacy perceived effectiveness of the behavior Bandura ${ }^{13}$ among the target audience, here the residents regarding following hand hygiene guidelines. Once a program is developed shown as in Table 4, it is highly recommended to have a kick-off meeting, preferably at lunchtime, for everyone involved. In this example, hospital executives and senior staff physicians should attend and explain their expectations of the residents, and promise to support residents' hand hygiene behavior. This meeting will dramatically improve residents' self-efficacy. In terms of response efficacy, the belief that "(washing hands) consumes too much time when using soap and water" is noticeable. In addition to system changes that make alcohol-based rubs more available, providing persuasive messages that alcohol-based rubs are as effective as soap and water, and that they save time will enhance response efficacy. Providing both self-efficacy and response efficacy messages along with programs handling environmental barriers will greatly enhance behavioral intention.

Table 4 Sample program based on the selected target beliefs

\section{Beliefs (or Bundle of beliefs) Program component example}

\begin{tabular}{|c|c|}
\hline & Emphasize this In the regularly education session. \\
\hline \multirow{2}{*}{$\begin{array}{l}\text { Protects resident (self) from } \\
\text { infection }\end{array}$} & Place posters where residents use, such as bathrooms, bedrooms. \\
\hline & $\begin{array}{l}\text { Discretion necessary; patients and families may perceive this message as hospitals staffs } \\
\text { consider them "dirty" and take offense to the message. Therefore, the venue to convey this } \\
\text { message had better be confined to the areas where only HCPs use. }\end{array}$ \\
\hline $\begin{array}{l}\text { Consumes too much time when } \\
\text { using soap and water }\end{array}$ & Advise residents to use alcohol-based rubs water \\
\hline Alcohol-based rubs water & $\begin{array}{l}\text { Find better placement for alcohol-based rubs Alcohol-based rubs are not around the ICUs; } \\
\text { shadowing residents is a good way to find the best places to place alcohol-based rubs. }\end{array}$ \\
\hline \multirow{2}{*}{$\begin{array}{l}\text { Makes skin too dry and sore on } \\
\text { resident' hands }\end{array}$} & Change current alcohol-based rub into more skin-friendly one. \\
\hline & Place a hand lotion near alcohol-based rubs. \\
\hline \multirow[b]{2}{*}{ I forget to wash my hands } & Place a warning sign such as "Did you wash your hands?" on the patient bedside. \\
\hline & $\begin{array}{l}\text { If enough resources are guaranteed, playing pre-recorded voice like "Did you wash your } \\
\text { hands?" whenever a person enters patient room. (In this case, senior staff's voice or hospital } \\
\text { executive's voice is recommended.) }\end{array}$ \\
\hline $\begin{array}{l}\text { Staff physicians (seniors) think } \\
\text { I should follow hand hygiene } \\
\text { guideline }\end{array}$ & $\begin{array}{l}\text { Hospital senior executive or director of ICUs have a meeting with senior staff physicians, and } \\
\text { explain how important residents' hand hygiene behavior depends on them: asking them to } \\
\text { support residents to wash hands regardless of how busy they are and to wash their hands too. }\end{array}$ \\
\hline $\begin{array}{l}\text { When seniors do not wash } \\
\text { their hands (for fear of looking } \\
\text { that the residents challenge the } \\
\text { seniors) }\end{array}$ & $\begin{array}{l}\text { An innovative approach in a hospital in US is that the president of the hospital directly } \\
\text { ordered medical school students doing clerkship to wash their hands; even nobody in the } \\
\text { patient room does perform hand hygiene behavior. The purpose of this is to breakdown } \\
\text { hierarchy that hampers HCPs' hand washing. Within a few weeks, everybody in the ICU } \\
\text { washes hands. }\end{array}$ \\
\hline
\end{tabular}

\section{Discussion}

This simple step-by-step guide was prepared to develop any programs for HCPs' behavioral change. People who are already familiar with the model used in this guideline may have questions. For example, we did not directly measure attitude, social norm and perceived behavioral control because identifying beliefs and following program development steps do not necessarily require them. Such thorough surveys usually take place for persuasive message development targeting a large population. In our case, and in many cases in health care field, the target audience has similar characteristics, especially when they are located in the same organization. They would share similar environment and cultural background. This would potentially result in much fewer behavior-relevant beliefs, which would make 
the statistical analyses manageable even when addressing all the key beliefs, without directly measuring attitude, social norm and perceived behavioral control and selecting one of the three before addressing each of the beliefs underlying those components. In addition we did not measure the residents' self-reported behavior, because the principal investigator began with secret shopper methods on HCPs' hand washing behavior. Therefore, the decision of success or failure of the project will rest on the next secret shopper measurement.

Though elicitation interview and calculating correlation between the belief and intention to perform the behavior are statistically clear processes, the step on "room for change" is based on the project manager's experience and decision. Indeed there is no clear rule that governs whether or not we choose a certain belief. What matters is filtering out the less important beliefs and the beliefs upon which we cannot develop an effective program. You may think of this process as ranking the beliefs, and take a few beliefs (in our example, seven were chosen) and take them to the program development step. If would rather limit yourself to three beliefs, just choose the three with high correlation and most room for change.

As shown in Table 4, one single belief can lead the program manager to devise multiple components of the program, and vice versa. As such, when developing a program, in contrast to the previous steps, you should be creative, and brainstorming is highly recommended.

In addition, when you follow this guideline, you may feel overwhelmed by the beliefs that emerge from the elicitation interviews. However daunting they look, all you need to do is develop and administer a sound survey to the target population and filter the results until you reach the addressable number of beliefs.

\section{Conclusion}

In conclusion, this guideline presents the statistical and solid steps to take to identify the key items that will lead a target population to perform a certain behavior. More importantly, this method can bring to light HCPs' highly relevant but latent beliefs. For example, many quality and patient safety leaders still believe that emphasizing "hand hygiene is important to save patients from infection" is crucial in improving HCPs' hand hygiene compliance. Though this belief is absolutely true, everybody already believes this, so focusing on this knowledge and educating the residents will not be very effective. As specialists in quality and safety, and communication science, the authors therefore suggest applying this step-by-step guide to any behavior-related problems in health care organization. We expect that this framework will have the desired results, and surely save lives.

\section{References}

1. Fishbein M, Ajzen I. Predicting and changing behavior: The reasoned action approach. USA: Psychology Press, New York; 2010. pp. 538.

2. Hornik R, Woolf KD. Using cross-sectional surveys to plan message strategies. Social Marketing Quarterly. 1999;5(2):34-41.

3. Wachter RM. Understanding patient safety. $2^{\text {nd }}$ edn. New York, USA: The McGraw-Hill Companies, Inc.; 2012.

4. Nicolay CR, Purkayastha S, Greenhalgh A, et al. Systematic review of the application of quality improvement methodologies from the manufacturing industry to surgical healthcare. Br J Surg. 2012;99(3):324335 .

5. Vest JR, Gamm LD. A critical review of the research literature on six sigma, lean and studergroup's hardwiring excellence in the united states: The need to demonstrate and communicate the effectiveness of transformation strategies in healthcare. Implement Sci. 2009;4: 35.

6. Jeong HJ, Pham JC, Kim M, et al. Major cultural-compatibility complex: Considerations on cross-cultural dissemination of patient safety programmes. BMJ Qual Saf. 2012;21(7):612-615.

7. Ajzen I. From intentions to actions: A theory of planned behavior. In: Kuhl J, Beckmann J, Editors. Action Control: From cognition to behavior. USA: Springer; 1985. pp. 286.

8. Fishbein M, Cappella JN. The role of theory in developing effective health communications. Journal of Communication. 2006;56(S1-S17).

9. Jeong HJ, Lee H, Lee W, et al. Attitudes toward, and facilitators/barriers for proper hand hygiene behavior amongst health care professionals in intensive care units of south korean hospitals: A qualitative study. France: Paper presented at the ISQua, Paris; 2010.

10. Fishbein M. The role of theory in HIV prevention. AIDS Care. 2000;12(3):273-278.

11. Montano DE, Kasprzyk D. The theory of reasoned action, theory of planned behavior and the integrated behavioral model. In: Glanz K, Editors, Health behavior and health education: Theory, research and practice, $4^{\text {th }}$ edn. San Francisco, USA: Jossey-Bass; 2008. pp. 67-96.

12. Yzer MC, Cappella JN, Fishbein M, et al. The effectiveness of gateway communications in anti-marijuana campaigns. J Health Commun. 2003;8(2):129-143.

13. Bandura A. Self-efficacy mechanism in human agency. American Psychologist. 1982;37(2):122-147. 